Onkologie 1992;15(suppl 1):1

\title{
Impressum, Vol. 15, Supplement 1, 1992
}

Ofrizielles Organ der Deutschen Gesellschaft für Hämatologie und Onkologie, der Osterreichischen Gesellschaft für Hämatologie und Onkologie, der Osterreichischen Krebsgesellschaft - Krebsliga unter Fortführung der «Österreichischen Zeitung für Onkologie» Schriftleitung

H. Huber, Innsbruck W. Queißer, Mannheim

Fachschriftleitung

W. E. Berdel, Berlin E. Dühmke, Göttingen N. Jaeger, Hildesheim M· Kaufmann, Heidelberg P. Schlag, Heidelberg Wissenschaftlicher Beirat

W. E. Berdel, Berlin V. Diehl, Köln

C. Dittrich, Wien

P. Drings, Heidelberg

E. Dühmke, Göttingen

H. H. Fiebig, Freiburg

A. Gläser, Halle

R. Hartenstein, München

K. Havemann, Marburg

K. P. Hellriegel, Berlin

R. Herrmann, Basel

H .W. von Heyden, Einbeck

D. Hoelzer, Frankfurt/M.

J. H. Holzner, Wien

H. Huber, Innsbruck H. J. Illiger, Oldenburg N. Jaeger, Hildesheim R. Jakesz, Wien M.

Kaufmann, Heidelberg

U. R. Kleeberg, Hamburg

B. Kornhuber, Frankfurt/M.

H. Löffler, Kiel

H. Ludwig, Wien

U. Mohr, Hannover

K. Munk, Heidelberg

G. A. Nagel, Vitikon-Zurich

A. Pñeiderer, Freiburg

F. Porzsolt, Ulm

K. Possinger, München

W Queißer, Mannheim

P. Schlag, Heidelberg

H.-J. Schmoll, Hannover

M. Schroder, Kassel

S. Seeber, Essen 
W. Vahlensieck, Bonn

H. W. Waclawiczek, Salzburg

M. Wannenmacher, Heidelberg

H. J. Weh, Hamburg

W. Wilmanns, München

\section{Anzeigen}

S. Karger Verlag für Medizin únd Naturwissenschaften GmbH, Postfach 1724, W-8034

Germering, Telefon (089) 843035. Gültig ist die Preisliste Nr. 6 vom 1.10.1990.

Für den Inhalt außerhalb des redaktionellen Teiles (insbesondere Anzeigen, Industrieinformationen, Pressezitate und Kongreßinforma-tionen usw.) übernehmen Schriftleitung, Beirat und Verlag keine Gewähr.

Eine Markenbezeichnung kann warenzeichengeschützt sein, auch wenn bei İhrer Verwendung in dieser Zeitschrift das Zeichen ${ }^{\circledR}$ oder ein anderer Hinweis auf etwa bestehende Schutzrechte fehlen sollte. Für Satzfehler, insbesondere bei Dosierungsangaben, wird keine Gewähr übernommen.

Alle Rechte, insbesondere das Recht der Vervielfáltigung und Mikro-kopie sowie der Übersetzung vorbehalten. Nachdruck, auch auszugs-weise, nur mit Genehmigung des Verlages. Fotokopien dürfen nur fur den persönlichen Gebrauch als Einzelkopien hergestellt werden. Jede im Bereich eines gewerblichen Unternehmens zulässig hergestellte oder benutzte Kopie dient gewerblichen Zwecken gem. § 54(2) UrhG und ver-pflichtet zur Gebührenzahlung an die Verwertungsgesellschaft WORT, Abt. VG Wissenschaft, Goethestr. 49, W-8000 München 2. (C) Copyright 1992 by S. Karger

Verlag für Medizin und Naturwissenschaften GmbH, Postfach 1724, W-8034 Germering (BRD)

ISBN 3-8055-5637-3

Verlagsleitung: Manfred R. Just Redaktionsassistenz: Anne Schieber Anzeigenverkauf: Marlene Scholz Anzeigenverwaltung: Eva M. Lindenau Herstellung: Horst H. Bruch Vertrieb: Gabriela Taube

Satz und Druck: Bonitas-Bauer, Grafischer Betrieb, Max-von-Laue-Str. 31, W-8700 Würzburg. inkl. MwSt.

Ladenpreis dieses Sonderheftes DM 28, Abonnenten erhalten es unberechnet.

Bezugspreis der Zeitschrift:

Für Jahrgang 14, 1991, DM 148,- /ÖS 1036,-/SFr 116,-.

1 Einzelheft kostet DM 28,-/öS 196,-/SFr 22,- einschließlich MwSt.

züglich Postgebühren.

KAKGEK 1992;015:1 